\title{
Circulating tumor cells (CTCs) in lung cancer: current status and future perspectives
}

\author{
This article was published in the following Dove Press journal: \\ Lung Cancer:Targets and Therapy \\ 3 July 2010 \\ Number of times this article has been viewed
}

\section{Fumihiro Tanaka \\ Kazue Yoneda \\ Seiki Hasegawa}

Department of Thoracic Surgery, Hyogo College of Medicine, Nishinomiya-city, Hyogo, Japan
Correspondence: Fumihiro Tanaka Department of Thoracic Surgery, Hyogo College of Medicine, Mukogawa I-I, Nishinomiya-city, Hyogo 663-850I, Japan Tel +8I 798456885

Fax +8I 798456897

Email ftanaka@hyo-med.ac.jp
Abstract: Primary lung cancer is the leading cause of cancer-related deaths in most industrialized countries, and it is important to detect and control metastases distant from the lungs for improvement of a patient's prognosis. Circulating tumor cells (CTCs) are tumor cells that are shed from the primary site and circulate in the peripheral blood, and recent studies have shown that CTCs can be useful clinical markers in some solid tumors such as those of breast cancer. In primary lung cancer, the clinical significance of CTCs remains unclear, but some promising results have been recently reported. Here, we reviewed the current status and future perspectives of CTCs in primary lung cancer.

Keywords: metastasis, cellsearch, CTC-chip, tumor cells

\section{Introduction}

Subdermal contraceptive implants have been studied and used in humans for over 20 years. ${ }^{1,2}$ The development of distant metastases is the most critical issue in treatment of patients with malignant tumors. It had been generally accepted that development of distant metastases was a late-stage event during the progression of solid malignant tumors, ${ }^{1}$ but recent experimental studies have revealed that tumor cells can spread to distant sites even at an early phase. ${ }^{2,3}$ In addition, recent clinical studies have revealed that tumor cells can be detected in the bone marrow and in the peripheral blood of patients with early-stage malignant tumors. ${ }^{4}$ Thus, detection of disseminated tumor cells (DTCs) in the bone marrow and circulating tumor cells (CTCs) in the peripheral blood can be clinically useful not only for early diagnosis of a malignant tumor but also for monitoring the therapeutic effect. In fact, many studies on the clinical significance of DTCs in a variety of malignant tumors showed that the presence of DTCs was significantly correlated with a poor prognosis. ${ }^{4-6}$ However, aspiration of the bone marrow for detection of DTCs is invasive and uncomfortable for patients. In contrast, sampling of the peripheral blood to detect CTCs can be easily and repeatedly performed, and is more preferable in clinical practice (Figure 1).

Primary lung cancer is the leading cause of cancer-related deaths in most industrialized countries. Approximately $40 \%$ of primary lung cancer patients initially present with distant metastases that are symptomatic or detectable with current diagnostic modalities including whole-body computed tomography (CT) and positron emission tomography (PET) scanning. ${ }^{7}$ More importantly, even in patients without clinically detectable distant metastasis at the time of initial diagnosis, distant metastases may frequently develop during treatment or long-term follow-up after 


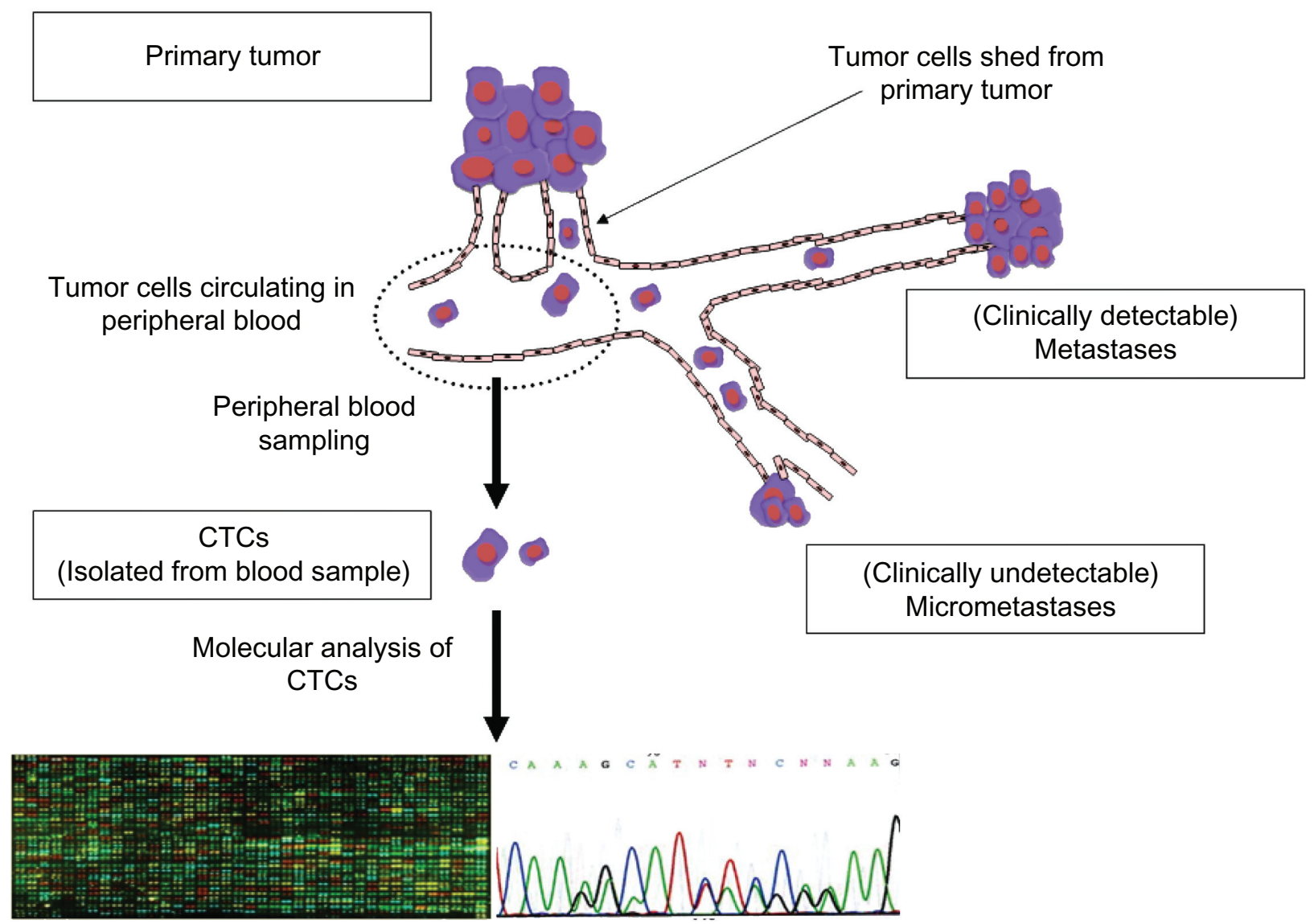

Figure I Sequence of the detection of circulating tumor cells (CTCs) and their molecular analysis. CTCs can be detected with direct or indirect methods. Those captured with direct methods can be saved for further analyses including molecular analysis.

treatment has been completed. Tumor cells may, therefore, circulate in the peripheral blood of most patients with primary lung cancer, which may cause the development of clinically apparent distant metastases. Thus, detection of such CTCs may contribute to improvement in diagnosis and therapy of primary lung cancer patients. ${ }^{2}$

In spite of many efforts to develop sensitive detection system of CTCs, these cells have not been established as clinical biomarkers mainly owing to a lack of reproducibility and accuracy in detection of CTCs. ${ }^{4}$ Recently, however, a semi-automated system for detection of CTCs, the CellSearch $^{\circledR}$ system (Veridex LLC, Raritan, NJ, USA) has been developed, ${ }^{8}$ and a CTC test with the CellSearch system has been approved by the Food and Drug Administration (FDA) in the USA for monitoring of blood from patients with metastatic breast, colon, and prostate carcinomas, which may provide a new insight into clinical usefulness of CTCs. Here, we review the current status and future perspectives of detection methods of CTCs and the potential clinical application of CTCs in the diagnosis and treatment of primary lung cancer patients.

\section{Detection methods of CTCs}

For detection of rare tumor cells circulating in the peripheral blood, a variety of methods have been developed, which are largely classified as either indirect or direct methods. Indirect methods, in which tumor-specific genes contaminating the peripheral blood were detected after amplification by polymerase chain reaction (PCR), have an advantage of higher sensitivity, but may be sometimes associated with unsatisfactory specificity and reproducibility. In contrast, direct methods have various advantages over indirect methods: they are able to provide morphological confirmation of CTCs, and they allow quantitative analysis of CTCs, and isolation of the cells for further analysis. Thus, direct methods have been predominantly employed in recent studies.

\section{Indirect methods}

Indirect detection methods generally comprise enriching the CTCs and then detecting a 'tumor-specific' marker gene by reverse transcription PCR (RT-PCR). The CTCenrichment step is important because CTCs are not abundant in the peripheral blood, being only a few cells 
per $10^{9}$ hematological cells. Enrichment methods include negative- or positive-selection methods using antibodies, density-gradient separation of mononuclear cells including tumor cells, and size-based separation. ${ }^{4,9}$ As 'tumor-specific' markers, epithelial-cell markers such as cytokeratin 19 (CK19) are most frequently used for indirect detection of $\mathrm{CTCs}^{10-16}$ (Table 1). Epithelial-cell markers are detectable not only in tumor cells originating from epithelial cells but also in normal epithelial cells. However, it is generally accepted that epithelial-cell markers are 'tumor-specific' markers for peripheral blood samples, because these markers are only detected in the peripheral blood when tumor cells of epithelial-cell origin are present.

Even though PCR-based techniques are very sensitive to epithelial-cell marker genes expressed in CTCs, these indirect methods are unfortunately also sensitive to epithelial-cell marker genes from skin cells that may have contaminated a sample as well as to low-level, spurious expression of nontumor cells. ${ }^{10,14,15}$ In addition, heterogeneity in the expression of marker genes means that a false-negative result may be expected with an indirect CTC test when tumor cells show minimal-level expression of epithelial-cell marker genes. ${ }^{14,16}$ To develop more sensitive and specific indirect methods of detection, other markers that are expressed at higher levels in tumor cells but not in normal cells have been examined, including carcinoembryonic antigen (CEA), ${ }^{14,17,18}$ squamous cell carcinoma antigen (SCC), ${ }^{13}$ and prepro-gastrin-releasing peptide (preproGRP). ${ }^{19}$ The detection of such 'tumor-specific' marker genes may provide higher specificity, but the expression of each marker gene usually tests positive in only a small subset of the entire population of primary lung cancer patients. For example, preproGRP is a useful marker only in small cell carcinoma (SCLC) patients, ie, only $10 \%-15 \%$ of all primary lung cancer patients. To overcome these issues, multiple markers including epithelial and 'tumor-specific' markers have been employed in some recent studies ${ }^{12,13}$ (Table 1).

\section{Direct methods}

Direct methods of detecting CTCs are superior to indirect ones because tumor cells circulating in the peripheral blood can be isolated, visualized, and saved for further analyses. However, isolation of rare CTCs dispersed among a large number of hematological cells presents a technical challenge. In fact, despite many efforts to develop a variety of direct methods such as size-selection methods, flow-cytometric methods, ${ }^{20}$ positive or negative immunomagnetic selection methods, and combinations of these, ${ }^{4,9}$ no direct CTC test has been established in clinical practice mainly owing to a lack of standardization of detection methods as well as a lack of reproducibility and accuracy in detection of CTCs. However, recent technological improvements have made it possible to isolate and quantitatively evaluate CTCs in the peripheral blood sampled from lung cancer patients ${ }^{21-27}$ (Table 2).

Table I Indirect methods for the detection of circulating tumor cells (CTCs) in primary lung cancer

\begin{tabular}{|c|c|c|c|c|}
\hline Markers & Reference & Positivity & Specificity & Comments \\
\hline \multicolumn{5}{|c|}{ Epithelial markers (cytokeratins) } \\
\hline \multirow[t]{4}{*}{ CKI9 } & 11 & $\begin{array}{l}37 \%(32 / 86): 40 \%(\mathrm{Ad}) ; 41 \%(\mathrm{Sq}) ; 27 \%(\mathrm{Sm}) \\
50 \% \text { (Stage I-II); 35\% (Stage III); } 41 \%(\text { Stage IV) }\end{array}$ & $98 \%(61 / 62)$ & \\
\hline & 12 & $41 \%(22 / 54)$ & $100 \%(24 / 24)$ & \\
\hline & 13 & $58 \%(78 / / 34)$ & $95 \%(176 / 186)$ & \\
\hline & 14 & ND & $0 \%(0 / 19)$ & \\
\hline \multirow[t]{2}{*}{ CK20 } & 14 & $0 \%(0 / 32)$ & $100 \%(19 / 19)$ & \\
\hline & 16 & $8 \%(1 / 13)$ & $100 \%(22 / 22)$ & \\
\hline \multicolumn{5}{|l|}{ Tumor-specific markers } \\
\hline \multirow[t]{2}{*}{ CEA } & 14 & $53 \%(17 / 32)$ & $100 \%(19 / 19)$ & \\
\hline & 17,18 & $60 \%(62 / 103)$ & $100 \%(47 / 47)$ & Correlation with survival \\
\hline SCC & 13 & $24 \%(32 / 134)$ & $97 \%(180 / 186)$ & \\
\hline \multirow[t]{2}{*}{ PreproGRP } & 13 & $34 \%(76 / 134)$ & $99 \%(185 / 186)$ & \\
\hline & 19 & $50 \%(16 / 32)$ of Sm: $58 \%$ (ED); 38\% (LD) & $100 \%(48 / 48)$ & \\
\hline \multicolumn{5}{|l|}{ Multiple markers } \\
\hline Four genes, including & 12 & $72 \%(39 / 54)$ & & Correlation with survival \\
\hline \multicolumn{5}{|l|}{ CKI9 } \\
\hline $\begin{array}{l}\text { BJ-TSA-9, Pre-proGRP, } \\
\text { KRT-19 }\end{array}$ & 13 & $\begin{array}{l}\text { At least one positive: } 84 \% \text { (II } 3 / / 34): 88 \%(\mathrm{Sq}) \text {; } \\
82 \% \text { (Ad); } 84 \% \text { (La \& Sm) }\end{array}$ & & $\begin{array}{l}\text { Correlation with stage } \\
\text { and survival }\end{array}$ \\
\hline
\end{tabular}

Abbreviations: CK, cytokeratin; Ad, adenocarcinoma; Sq, squamous cell carcinoma; Sm, small cell carcinoma; ND, not determined; ED, extended disease; LD, limited disease; La, large cell carcinoma. 
Table 2 Direct methods for detection of circulating tumor cells (CTCs) in primary lung cancer

\begin{tabular}{|c|c|c|c|c|c|}
\hline Method and study & Patients & Positivity & CTC count & Specificity & Comments \\
\hline \multicolumn{6}{|c|}{ Cytelligen: Negative immunomagnetic selection with anti-CD45 Ab followed by CK8/I 8// 9 immunostaining } \\
\hline \multirow[t]{4}{*}{$\mathrm{Wu}^{21}$} & SCLC and NSCLC & $85 \%(35 / 4 I)$ & & $90 \%(28 / 31)$ & \\
\hline & & $(\mathrm{CTC} \geq \mathrm{I})$ & & $(\mathrm{CTC} \geq \mathrm{I})$ & \\
\hline & & $83 \%$ & & $97 \%(30 / 31)$ & \\
\hline & & $(\mathrm{CTC} \geq 2)$ & & $(\mathrm{CTC} \geq 2)$ & \\
\hline
\end{tabular}

MAINTRAK: Positive immunomagnetic selection with anti-epithelial Ab followed by laser scanning cytometry

Rolle $^{22}$ NSCLC (Stage I-III) $\quad 86 \%$

$(25 / 29)$

CellSearch: Positive immunomagnetic selection with anti-EpCAM Ab followed by morphological confirmation with staining for cytokeratins (positive in cytoplasm), DAPI (positive in nucleus), and CD45 (negative)

Sawabata ${ }^{23}$

$\mathrm{Hou}^{24}$

NSCLC (Stage I)
SCLC

CTC count

decreased with chemotherapy *Prognostic (univariate)

Tanaka ${ }^{25} \quad$ SCLC and NSCLC CTC-chip: Capture with microposts coated with anti-EpCAM antibodies Nagrath $^{26} \quad$ NSCLC $\quad 100 \%(55 / 55) \quad 155$ cells $/ 1.0 \mathrm{~mL}$ (mean) (5-128I)

EGFR-gene mutations detected from $\mathrm{CTCs}^{27}$

Abbreviations: CK, cytokeratin; Ab, antibody; SCLC, small cell lung cancer; NSCLC, non-small cell lung cancer; EpCAM, epithelial cell adhesion molecule; ED, extended disease; LD, limited disease; EGFR, epithelial growth factor receptor.

Of the direct detection methods employed, the CellSearch system is the one most commonly used in clinical studies. ${ }^{8,23-27}$ In this system, an antibody against the epithelial cell adhesion molecule (EpCAM) is used to capture CTCs, as the EpCAM is a pan-epithelial marker as well as a tumor-associated marker (also known as 'tumor-associated calcium signal transducer 1') and is abundantly expressed on the surface of tumor cells of epithelial-cell origin. The most important advantage of the CellSearch system is reproducibility across different laboratories, ${ }^{28}$ which has generally been achieved with semi-automated isolation and morphological verification of CTCs. More specifically, CTCs were automatically isolated from a $7.5 \mathrm{~mL}$ of blood with immunomagnetic capture by ferroparticles coupled to an anti-EpCAM antibody, and were analyzed on a florescent microscope using the Cell Track Analyzer after staining with a mixture of phycoerythrin-conjugated anti-CK8/18/19 antibodies, an allophycocyanin-conjugated anti-CD45 antibody, and nuclear dye 4',6-diamidino-2-phenylindole (DAPI). The criteria for each cell to be defined as a CTC are as follows: round to oval morphology, a visible DAPI-positive nucleus present in CK-positive cytoplasm, and negative staining for CD45 (Figure 2). In our prospective study, ${ }^{25} \mathrm{CTCs}$ were identified in $38(30.6 \%)$ of 125 primary lung cancer patients; CTCs were also identified in $3(12.0 \%)$ of 25 patients with nonmalignant lung diseases, but the CTC count was only 1 or 2 cells $/ 7.5 \mathrm{~mL}$ (Figure 3A). CTC counts were significantly higher in lung cancer patients than in those with nonmalignant diseases, but receiver operating characteristic (ROC) curve analysis failed to show a significant diagnostic value for the CTC test in discriminating between the two patient categories (Figure 3B). Among lung cancer patients, CTC counts were significantly higher in SCLC patients than in non-SCLC (NSCLC) patients (Figure 4A). More importantly, CTC count significantly increased with tumor progression and according to ROC curve analysis, the diagnostic performance of the CTC test (predicting development of distant metastasis) was significant (Figure 5). Thus, using the CTC test in conjunction with the CellSearch system might be clinically useful in the diagnosis of metastatic lung cancer, but the sensitivity remains unsatisfactory because $29.0 \%$ of stage IV patients showed a negative CTC count (' 0 ') although distant metastasis was detected with routine clinical testing, including whole-body CT and PET scanning.

As discussed in the previous section, it is generally accepted that epithelial cells present in the peripheral blood are tumor cells, and epithelial cells captured with an anti-EpCAM antibody in the CellSearch system can be considered to be CTCs. 


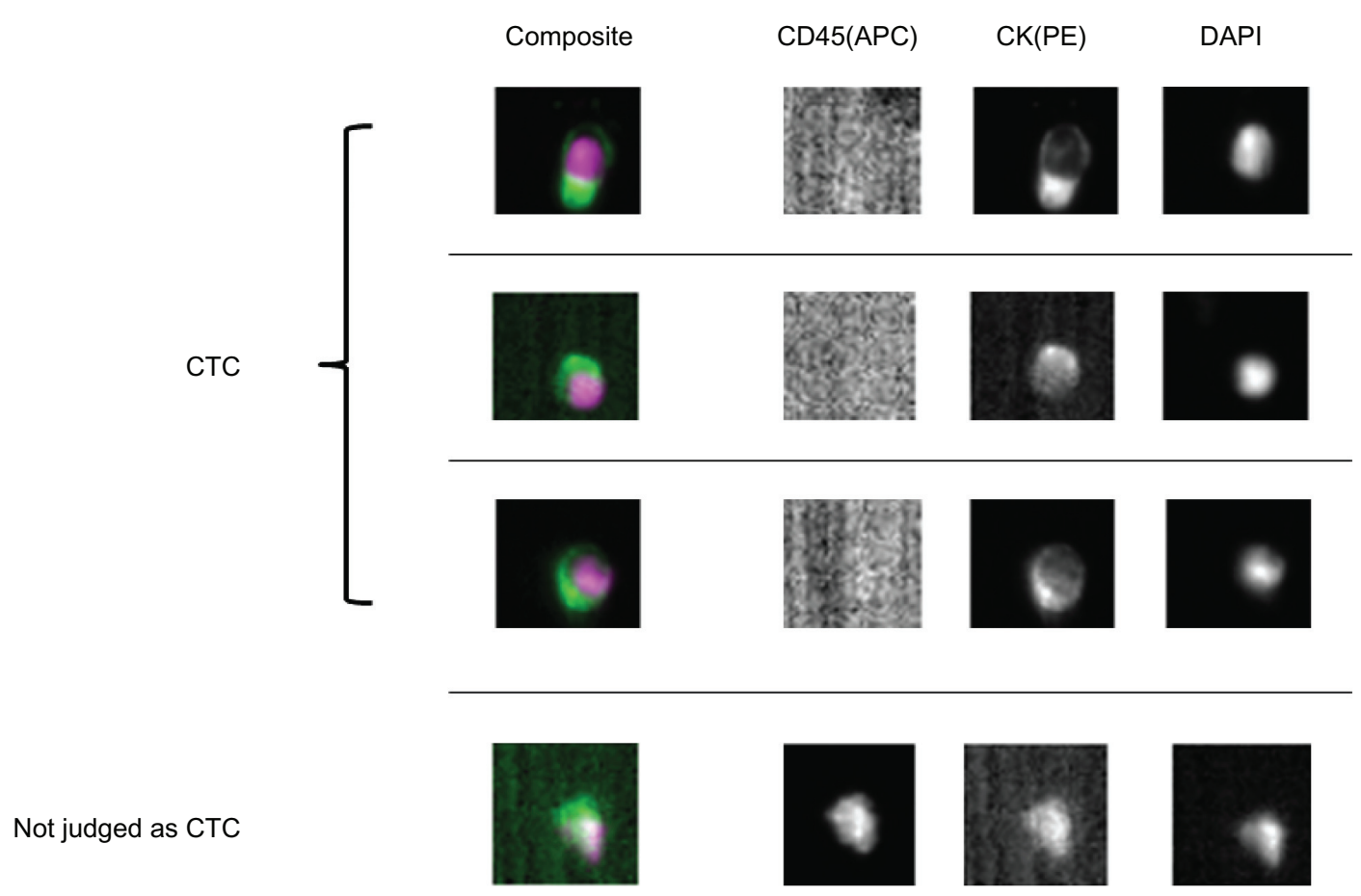

Figure 2 Circulating tumor cells (CTCs) captured with the CellSearch system from peripheral blood sampled from primary lung cancer patients. CTCs are stained with anti-CD45-allophycocyanin (CD45(APC)), anti-cytokeratin-phycoerythrin (CK(PE)) and 4',6-diamino-2-phenylindole (DAPI). Cells with round to oval morphology, a visible DAPI-positive nucleus, positive CK-PE staining in the cytoplasm, and negative staining for CD45-APC were considered to be CTCs. Contaminated white blood cells (WBCs) were excluded by CD45-APC staining.

However, it is still unclear whether epithelial cells defined as 'CTCs' are true tumor cells and/or whether these 'CTCs' actually grow at distant organs to form metastatic foci. In fact, in our study, ${ }^{25}$ CTCs tested positive in 3 of 25 patients with nonmalignant diseases, and such false-positive results may be caused by 1) contamination of epithelial cells in blood samples due to a variety of technical issues such as inappropriate blood sampling, 2) false-positive staining of contaminated nonepithelial cells for cytokeratin/DAPI during sample processing, 3) inappropriate judgment in the identification of CTCs by researchers.

The CTC-chip system is a novel microfluidic platform for detecting CTCs, which consists of an array of 78,000 microposts
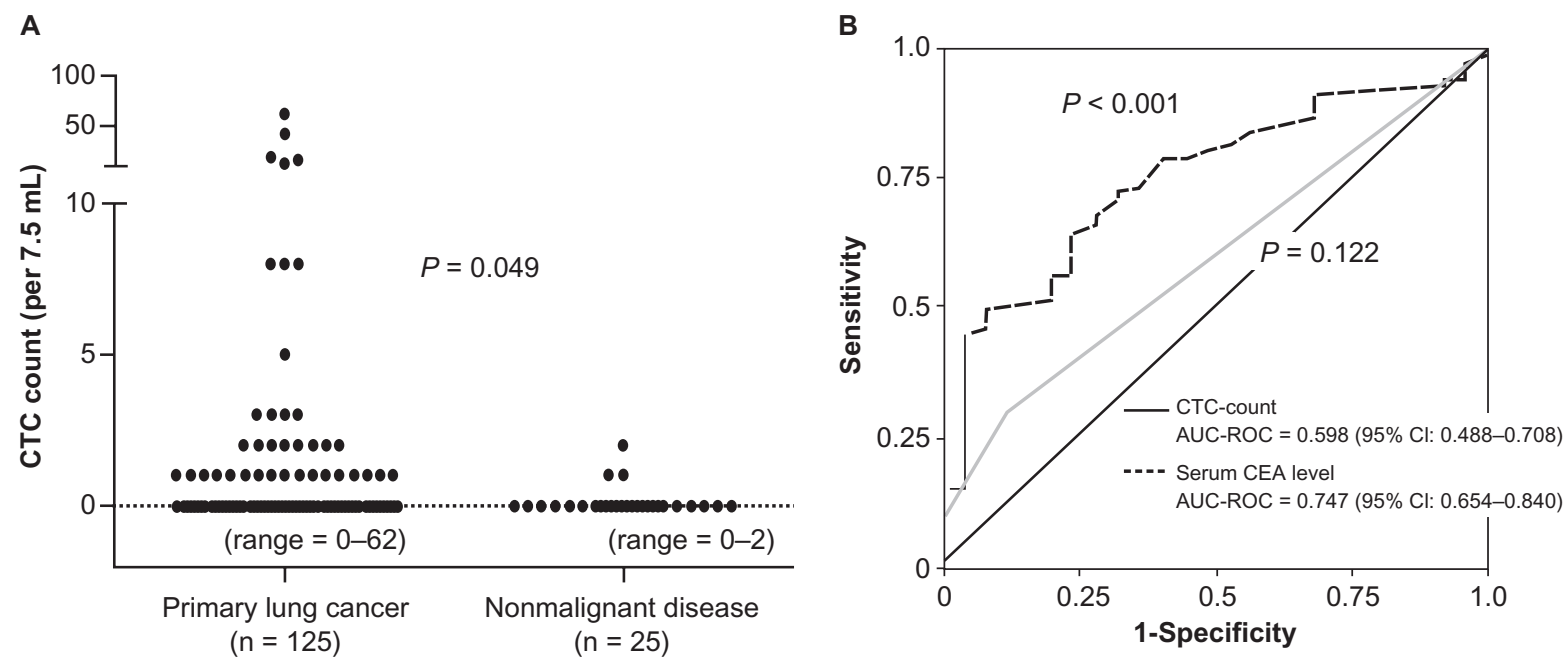

Figure 3 A) Distribution of circulating tumor cell (CTC) counts in $7.5 \mathrm{~mL}$ of peripheral blood sampled from primary lung cancer patients and patients with nonmalignant lung diseases. CTCs were isolated and quantitatively evaluated with the CellSearch system. B) Receiver operating characteristic (ROC) curves for CTC counts and serum Carcinoembryonic Antigen (CEA) levels for discriminating primary lung cancer patients from those with nonmalignant diseases. Figures taken from Tanaka et al. ${ }^{25}$ 

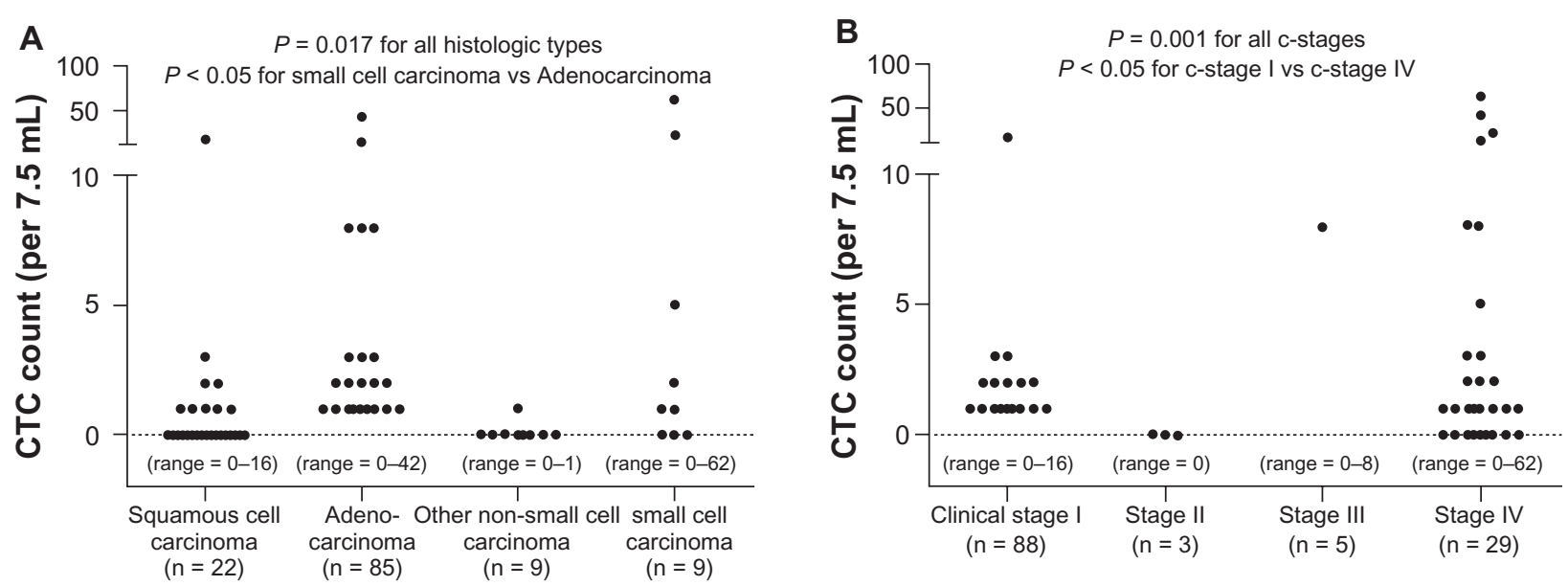

Figure 4 A) Distribution of circulating tumor cell (CTC) counts in $7.5 \mathrm{~mL}$ of peripheral blood sampled from primary lung cancer patients according to histologic cell type. B) Distribution of (CTC) counts in $7.5 \mathrm{~mL}$ of peripheral blood sampled from primary lung cancer patients according to clinical (c-) stage. Figures taken from Tanaka et al. ${ }^{25}$

coated with anti-EpCAM antibodies, which capture the CTCs under laminar flow conditions. ${ }^{26,27}$ The CTC-chip system may provide higher sensitivity in identification of CTCs than the method described above. In fact, a pilot study conducted by Nagrath and coworkers showed that CTCs were detected in all peripheral blood sampled from NSCLC patients and that CTC counts were higher (mean CTC count $=155$ cells $/ 1.0 \mathrm{~mL}$ ) as compared with those yielded with the CellSearch system. ${ }^{27}$

\section{Clinical relevance of CTCs}

The clinical value of CTCs in the diagnosis and therapy of primary lung cancer patients is yet to be established, but CTCs may be useful clinical markers for resectable and un-resectable diseases.

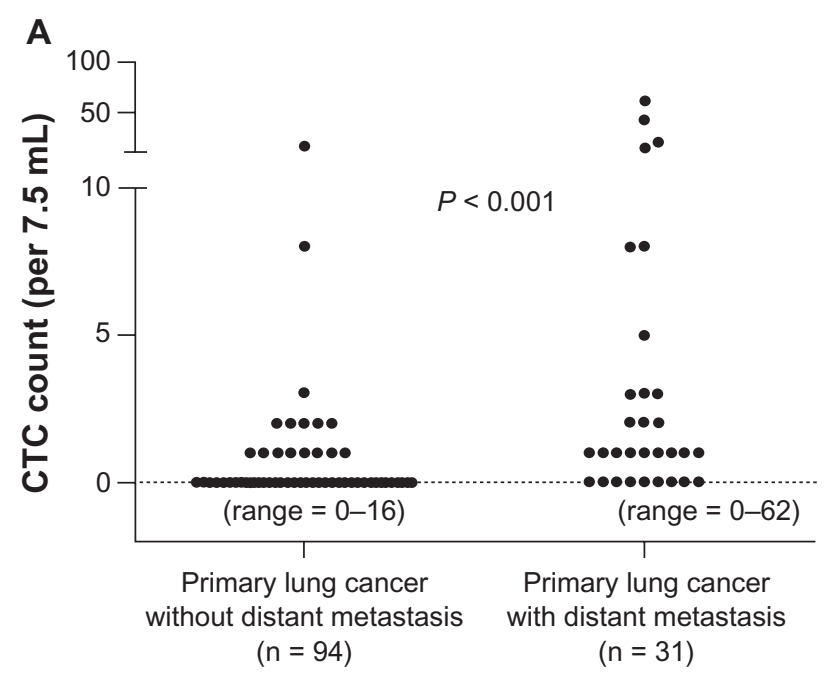

\section{Resectable disease}

Primary lung cancer is generally classified into SCLC or NSCLC, the latter including squamous cell carcinoma, adenocarcinoma, and large cell carcinoma. ${ }^{29-32}$ Most SCLC patients cannot be candidates for surgical resection because of early development of nodal and/or distant metastases. ${ }^{33,34}$ For patients with NSCLC, who account for $80 \%-85 \%$ of primary lung cancer, surgery is the treatment of choice. However, even when complete resection is achieved, postoperative prognosis remains poor, with 5-year overall survival rates of $60 \%-70 \%$ for pathologic (p-) stage-I, 40\%-50\% for p-stage-II, and $20 \%-30 \%$ for p-stage III patients. ${ }^{29-32,35,36}$ To improve the prognosis, postoperative adjuvant chemotherapy has been now established as a standard therapy based on documented evidence

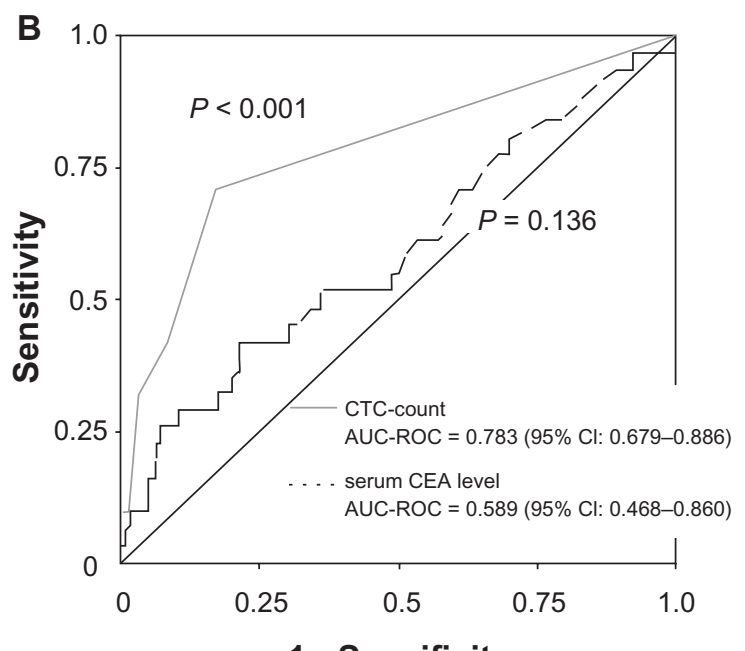

\section{1 - Specificity}

Figure 5 A) Distribution of circulating tumor cell (CTC) counts in $7.5 \mathrm{~mL}$ of peripheral blood sampled from primary lung cancer patients with and without distant metastasis (metastatic lung cancer and nonmetastatic lung cancer, respectively). B) Receiver operating characteristic (ROC) curves for CTC counts and serum Carcinoembryonic Antigen (CEA) levels for discriminating metastatic lung cancer patients from nonmetastatic lung cancer. Figures taken from Tanaka et al. ${ }^{25}$ 
from a number of clinical trials. ${ }^{37}$ Among stage I patients, those with earlier stage of the disease (stage IA) show a favorable prognosis with surgery alone, and no adjuvant chemotherapy is recommended; for patients with stage IB disease, tegafur uracil (UFT), an oral chemotherapeutic agent developed in Japan, is effective and is frequently prescribed in Japan. ${ }^{38}$ For patients with more advanced diseases such as stage II and III diseases, cisplatin-based intravenous chemotherapy is recommended after complete resection. ${ }^{39}$ However, if all tumor cells are removed with surgery, no recurrence occurs in any patient, and postoperative adjuvant chemotherapy is essentially 'unnecessary' and can be harmful. ${ }^{40}$ Thus, the presence of minimal residual tumors such as micro-metastases, which cannot be detected clinically, may be responsible for tumor recurrence after complete resection. One potential advantage of a CTC test may be its ability to predict the presence of a micro-metastasis that is undetectable with routine diagnostic modalities. In our prospective study, CTCs were detected in 17 (19.3\%) of 88 clinical (c-) stage I patients, and the presence of distant metastasis (all intrapulmonary metastasis) was confirmed in resected specimens in 2 of 17 CTC-positive c-stage I patients. ${ }^{25}$ Further long-term follow-up of the remaining 15 patients with and without CTC-positive, documented metastatic disease at the time of surgery will reveal whether CTCs detected in these patients are correlated with the development of micrometastasis causing postoperative recurrence. When future experimental and clinical studies reveal that CTCs can be surrogates of micro-metastasis, tests for the presence of these cells can be used to aid decisions about using adjuvant chemotherapy for completely resected patients. More specifically, CTC tests can be used to distinguish between a completely resected patient that is curable with surgery alone and a patient with a minimal residual tumor whose cure necessitates adjuvant chemotherapy.

\section{Unresectable disease}

Systemic chemotherapy is usually performed for unresectable lung cancer patients with good organ function, and CTCs may be a useful marker not only in predicting their prognosis but also in monitoring the therapeutic effect. In addition, molecular characteristics of CTCs may provide useful information for treatment of lung cancer patients (Figure 1). Maheswaran and coworkers reported that mutations in the epidermal growth factor receptor (EGFR) gene can be detected in CTCs isolated from lung cancer patients. ${ }^{27}$ As the presence of activated mutations in the tyrosine kinase domain of the EGFR gene is the strongest predictor for a favorable response to EGFR-tyrosine kinase inhibitors (TKIs) such as gefitinib and erlotinib, ${ }^{39,40}$ detection of EGFR mutations is essential in decisions about the treatment for unresectable lung cancer patients. ${ }^{41}$ However, it is sometimes difficult to obtain tumor cells suitable for gene mutation analysis from advanced lung cancer patients by routine biopsy modalities such as transbronchial biopsy under bronchoscopy and percutaneous needle biopsy. Molecular analysis of CTCs can be performed noninvasively and repeatedly, and it reveals not only EGFR mutational status (thereby allowing prediction of the response to EGFR-TKIs) but also whether EGFR mutations related to resistance during treatment with EGFR-TKIs have developed.

\section{Conclusion}

CTCs may be useful biomarkers in the diagnosis and therapy of primary lung cancer patients. Further experimental and clinical studies are warranted to determine the nature of CTCs as well as their clinical value.

\section{Acknowledgments}

This study is supported by the Grants-in-Aid 20390374 (F Tanaka) for Scientific Research (B) from the Japan Society for the Promotion of Science, Japan. We thank Mrs Mayo Yamamoto for helpful assistance in the preparation of the manuscript.

\section{Disclosure}

The authors report no conflicts of interest in this work.

\section{References}

1. Eccles SA, Welch DR. Metastasis: recent discoveries and novel treatment strategies. Lancet. 2007;369(9574):1742-1757.

2. Husemann Y, Geigl JB, Schbert F, et al. Systemic spreads in an early step in breast cancer. Cancer Cell. 2008;13(1):58-68.

3. Klein CA. The metastasis cascade. Science. 2008;321:1785-1787.

4. Alix-Panabieres C, Riethdorf S, Pantel K. Circulating tumor cells and bone marrow micrometastasis. Clin Cancer Res. 2008;14(16):5013-5021.

5. Pantel K, Cote RJ, Fodstad O. Detection and clinical importance of micrometastatic disease. J Natl Cancer Inst. 1999;91(13):1113-1124.

6. Riethdorf S, Pantel K. Disseminated tumor cells in bone marrow and circulating tumor cells in blood of breast cancer patients: current state of detection and characterization. Pathology. 2008;75(2):140-148.

7. Socinski MA, Crowell R, Hensing TE, Langer CJ, Lielenbaum R, Sandler AB, Morris D. Treatment of non-small cell lung cancer, stage IV: ACCP evidence-based clinical practice guidelines (2nd edition). Chest. 2007;132 Suppl 3:S277-S289.

8. Allard WJ, Matera J, Miller MC, et al. Tumor cells circulate in the peripheral blood of all major carcinomas but not in healthy or patients with nonmalignant diseases. Clin Cancer Res. 2004;10(20):6897-6904.

9. Pantel K, Brakenhoff RH, Brandt B. Detection, clinical relevance and specific biological properties of disseminated tumor cells. Nat Rev Cancer. 2008;8(5):329-340.

10. Xi L, Nicastri DG, El-Hefnawy T, Hughes SJ, Luketich JD, Godfrey TE. Optimal markers for real-time quantitative reverse transcription PCR detection of circulating tumor cells from melanoma, breast, colon, esophageal, head and neck, and lung cancers. Clin Chem. 2007;53(7): 1206-1215. 
11. Peck K, Sher YP, Shih JY, Roflfer SR, Wu CW, Yang PC. Detection and quantitation of circulating cancer cells in the peripheral blood of lung cancer patients. Cancer Res. 1998;58(13):2761-2765.

12. SherYP, Shih JY, Yang PC, et al. Prognosis of non-small cell lung cancer patients by detecting circulating tumor cells in the peripheral blood with multiple marker genes. Clin Cancer Res. 2005;11(1):173-179.

13. Liu L, Liao GQ, He P, et al. Detection of circulating cancer cells in lung cancer patients with a panel of marker genes. Biochem Biophys Res Commun. 2008;372(4):756-760.

14. Castaldo G, Tomaiuolo R, Sanduzzi A, Ponticiello A, Marchetiello I, Salvatore F. Carcinoembryonic antigen mRNA analysis detects micrometastatic cells in blood from lung cancer patients. Eur Respir J. 2003; 22(3):418-421.

15. Dingemans AM, Brakenhoff RH, Postmus PE, Giaccone G. Detection of cytokeratin-19 transcripts by reverse transcriptase-polymerase chain reaction in lung cancer cell lines and blood of lung cancer patients. Lab Invest. 1997;77(3):213-220.

16. Chausovsky G, Luchansky M, Figer A, et al. Expression of cytokeratin 20 in the blood of patients with disseminated carcinomas of the pancreas, colon, stomach, and lung. Cancer. 1999;86(11):2398-2405.

17. Kurusu Y, Yamashita J, Hayashi N, Mita S, Fujino N, Ogawa M. The sequence of vessel ligation affects tumor release into the circulation. J Thorac Cardiovasc Surg. 1998;116(1):107-113.

18. Yamashita J, Matsuo A, Kurusu Y, Saishoji T, Hayashi N, Ogawa M. Preoperative evidence of circulating tumor cells by means of reverse transcriptase-PCR for carcinoembryonic antigen messenger RNA is an independent predictor of survival in non-small cell lung cancer: a prospective study. J Thorac Cardiovasc Surg. 2002;124(2):299-305.

19. Saito T, Kobayashi M, Harada R, Uemura Y, Taguchi H. Sensitive detection of small cell lung carcinoma cells by reverse transcriptasepolymerase chain reaction for prepro-gastrin-releasing peptide mRNA. Cancer. 2003;97(10):2504-2511.

20. Kularantne BY, Lorigan P, Browne S, Suvama SK, Smith MO, Lawry J. Monitoring tumor cells in the peripheral blood of small cell lung cancer patients. Cytometry. 2002;50(3):160-167.

21. Wu C, Hao H, Li L, et al. Preliminary investigation of the clinical significance of detecting circulating tumor cells enriched from lung cancer patients. $J$ Thorac Oncol. 2009;4(1):30-36.

22. Rolle A, Gunzel R, Pachmann U, Willen B, Hoffken K, Pachmann K. Increase in number of circulating disseminated epithelial cells after surgery for non-small cell lung cancer monitored by MAINTRAC is a predictor for relapse: a preliminary report. World J Surg Oncol. 2005;3(1):18.

23. Sawabata N, Okumura M, Utsumi T, et al. Circulating tumor cells in peripheral blood caused by surgical manipulation of non-small-cell lung cancer: pilot study using an immunocytology method. Gen Thorac Cardiovasc Surg. 2007;55(5):189-192.

24. Hou JM, Greystoke A, Lancashire L, et al. Evaluation of circulating tumor cells and serological cell death biomarkers in small cell lung cancer patients undergoing chemotherapy. Am J Pathol. 2009;175(2): 808-816.

25. Tanaka F, Yoneda K, Kondo N, et al. Circulating tumor cell as a diagnostic marker in primary lung cancer. Clin Cancer Res. 2009; 15:6980-6986.

26. Nagrath S, Sequist LV, Maheswaran S, et al. Isolation of rare circulating tumor cells in cancer patients with microchip technology. Nature. 2007;450(7173):1235-1239.
27. Maheswaran S, Sequist L, Nagrath S, et al. Detection of mutations in EGFR in circulating lung-tumor cells. N Engl J Med. 2008;359(4): 366-377.

28. Riethdort S, Fritsche H, Muller V, et al. Detection of circulating tumor cells in peripheral blood of patients with metastatic breast cancer: a validation study of the CellSearch system. Clin Cancer Res. 2007;13(3): 920-928.

29. Mountain CF. Revisions in the International system for staging lung cancer. Chest. 1997;111(6):1710-1717.

30. Koike T, Yamato Y, Asamura H, et al. Improvements in surgical results for lung cancer from 1989-1999 in Japan. J Thorac Oncol. 2009;4(11):1364-1369.

31. Asamura H, Goya T, Koshiishi Y, et al. A Japanese Lung Cancer Registry study: prognosis of 13,010 resected lung cancers. $J$ Thorac Oncol. 2008;3(1):46-52.

32. Goldstraw P, Crowly J, Chansky K, et al. The IASLC lung cancer project: proposals for the revision of the TNM stage groupings in the forthcoming (seventh) edition of the TNM classification of malignant tumors. J Thorac Oncol. 2007;2(8):706-714.

33. Shepherd FA, Crowly J, van Hotte P, et al. The International Association for the Study of Lung Cancer lung cancer staging project: proposals regarding the clinical staging of small cell lung cancer in the forthcoming (seventh) edition of the tumor, node, metastasis classification for lung cancer. $J$ Thorac Oncol. 2007;2(12):1067-1077.

34. Vallieres E, Shepherd FA, Crowly J, et al. The IASLC lung cancer staging project: proposal regarding the relevance of TNM in the pathologic staging of small cell lung cancer in the forthcoming (seventh) edition of the TNM classification for lung cancer. J Thorac Oncol. 2009;4(9): 1049-1059.

35. Tanaka F, Yanagihara K, Otake Y, et al. Surgery for non-small cell lung cancer: postoperative based on the revised tumor-node-metastasis classification and its time trend. Eur J Cardiothorac Surg. 2000; 18(2):147-155.

36. Chansky K, Sculier JP, Crowly JJ, Giroux D, van Hotte J, Goldstraw P. The International Association for the Study of Lung Cancer staging project: prognostic factors and pathologic TNM stage in surgically managed non-small cell lung cancer. J Thorac Oncol. 2009;4(7):792-801.

37. Pisters KMW, Evans WK, Azzoli CG, et al. Cancer Care Ontario and American Society of Clinical Oncology adjuvant chemotherapy and adjuvant radiation therapy for stage I-IIIa resectable non-small cell lung cancer guideline. J Clin Oncol. 2007;25:5506-5518.

38. Hamada C, Tanaka F, Ohta M, et al. Meta-analysis of postoperative adjuvant chemotherapy with tegafur-uracil in non-small cell lung cancer. J Clin Oncol. 2005;23(22):4999-5006.

39. Pignon JP, Tribodet H, Scagliotti GV, et al. Lung adjuvant cisplatin evaluation: a pooled analysis by the LACE collaborative group. J Clin Oncol. 2008;26(21):3552-3559.

39. Mok T, Wu YL, Thongprasert S, et al. Gefitinib or carboplatin-paclitaxel in lung adenocarcinoma. $N$ Engl J Med. 2009;361(10):947-957.

40. Mitsudomi T, Morita S, Yatabe Y, et al. Gefitinib versus cisplatin plus docetaxel in patients with non-small cell lung cancer harbouring mutations of the epidermal growth factor receptor (WJTOG3405): an open label, randomized phase 3 trial. Lancet Oncol. 2010;11(2):121-128.

41. Rosell R, Viteri S, Molina MA, Benilloch S, Taron M. Epidermal growth factor receptor tyrosine kinase inhibitors as first-line treatment in advanced non-small cell lung cancer. Curr Opin Oncol. 2010;22(2): 112-120.

Lung Cancer: Targets and Therapy

\section{Publish your work in this journal}

Lung Cancer: Targets and Therapy is an international, peer-reviewed, open access journal focusing on lung cancer research, identification of therapeutic targets and the optimal use of preventative and integrated treatment interventions to achieve improved outcomes, enhanced survival and quality of life for the cancer patient. Specific topics covered in the journal include:

Epidemiology, detection and screening; Cellular research and biomarkers; Identification of biotargets and agents with novel mechanisms of action; Optimal clinical use of existing anticancer agents, including combination therapies; Radiation and surgery; Palliative care; Patient adherence, quality of life, satisfaction; Health economic evaluations. 